VERSITA

\author{
TATRA \\ MOUNTaiNs \\ Mathematical Publications \\ DOI: $10.2478 / \mathrm{v} 10127-011-0015-3$ \\ Tatra Mt. Math. Publ. 48 (2011), 153-163
}

\title{
OSCILLATORY CRITERIA FOR TWO-DIMENSIONAL SYSTEM OF DIFFERENCE EQUATIONS
}

\author{
ZDENĚK OPLUŠTIL
}

\begin{abstract}
Some oscillation criteria are established for two-dimensional systems of first order linear difference equations.
\end{abstract}

\section{Introduction and notation}

This paper is devoted to the oscillatory properties of two-dimensional system of linear difference equations

$$
\begin{aligned}
\Delta u_{k} & =q_{k} v_{k}, \\
\Delta v_{k} & =-p_{k} u_{k+1},
\end{aligned}
$$

where

$$
\Delta x_{k}=x_{k+1}-x_{k}, \quad p_{k}, q_{k} \in R \quad \text { for } \quad k \in \mathbb{N} .
$$

System (11) is one of the possible discrete analogies of the linear Hamiltion system of differential equations

$$
\begin{aligned}
& u^{\prime}=q(t) v, \\
& v^{\prime}=-p(t) u,
\end{aligned}
$$

where $p(t), q(t)$ are continuous functions defined on $\left[t_{0},+\infty\right)$.

By a solution of system (11) we understand a vector sequence $\left\{\left(u_{k}, v_{k}\right)\right\}_{k=1}^{+\infty}$ satisfying system (1) for every natural $k$. A nontrivial solution $\left\{\left(u_{k}, v_{k}\right)\right\}_{k=1}^{+\infty}$ of system (11) is said to be oscillatory if there exists an infinite set $N_{0} \subseteq N$ such that

$$
u_{k} u_{k+1} \leq 0 \quad \text { for } \quad k \in N_{0}
$$

(C) 2011 Mathematical Institute, Slovak Academy of Sciences. 2010 Mathematics Subject Classification: 39A21.

Keywords: two dimensional system, linear difference equation, oscillatory solution.

Supported by Grant No. FSI-S-10-14 "Modern methods of mathematical problem modelling in engineering" and research plan MSM 0021630518 "Simulation modelling of mechatronic systems of the Ministry of Education, Youth and Sports of the Czech Republic. 


\section{ZDENĚK OPLUŠTIL}

It is known (see, e.g., [1]) that if

$$
q_{k} \geq 0 \quad \text { for } \quad k \in N
$$

and system (11) has at least one oscillatory solution, then all its solutions are oscillatory. That is why we can introduce the following definition.

Definition 1.1. System (11) is said to be oscillatory if all its solutions are oscillatory, otherwise (11) is said to be non-oscillatory.

Oscillatory properties of system (11) are known in the case where

$$
0<m \leq q_{k} \quad \text { for } \quad k \in \mathbb{N} \quad \text { and } \quad \sum_{j=1}^{+\infty} p_{j}=+\infty
$$

hold (see, e.g., [1]) or in the case where following conditions

$$
0<m \leq q_{k} \quad \text { for } \quad k \in \mathbb{N} \quad \text { and } \quad-\infty=\liminf _{k \rightarrow \infty} \sum_{j=1}^{k} p_{j}<\limsup _{k \rightarrow \infty} \sum_{j=1}^{k} p_{j},
$$

are fulfilled. System (11) is oscillatory in both mentioned cases.

In this paper sufficient conditions guaranteeing that the system (11) is oscillatory are established for cases where the series $\sum_{j=1}^{+\infty} p_{j}$ converges to a finite number, i.e.,

$$
\left|\sum_{j=1}^{+\infty} p_{j}\right|<+\infty
$$

and

$$
0<m \leq q_{k} \leq M<+\infty \quad \text { for } \quad k \in N,
$$

where $m, M$ are real positive constants.

In what follows we will suppose that there exists a finite limit

$$
c_{0}=\lim _{k \rightarrow \infty} c_{k},
$$

where

$$
c_{k}=\frac{1}{\sum_{j=1}^{k-1} q_{j}} \sum_{j=1}^{k-1} q_{j} \sum_{i=1}^{j-1} p_{i} \quad \text { for } \quad k \in N .
$$

Let us introduce following notations

$$
Q_{k}=\left(c_{0}-\sum_{j=1}^{k-1} p_{j}\right) \sum_{j=1}^{k-1} q_{j}=\sum_{j=1}^{k-1} q_{j} \sum_{j=k}^{\infty} p_{j} \quad \text { for } \quad k \in \mathbb{N}
$$




$$
\begin{gathered}
H_{k}=\frac{1}{\sum_{j=1}^{k-1} q_{j}} \sum_{j=1}^{k} p_{j}\left(\sum_{i=1}^{j} q_{i}\right)^{2} \quad \text { for } \quad k \in \mathbb{N}, \\
Q_{*}=\liminf _{k \rightarrow \infty} Q_{k}, \quad Q^{*}=\limsup _{k \rightarrow \infty} Q_{k}, \\
H_{*}=\liminf _{k \rightarrow \infty} H_{k}, \quad H^{*}=\limsup _{k \rightarrow \infty} H_{k} .
\end{gathered}
$$

\section{Main results}

Below formulated theorems generalize and make more complete previous well-known criteria of analogous types. Presented results can be understood as a difference analogy of oscillatory theorems for ordinary differential equations which can be found in [3]-[5].

Theorem 2.1. Let

$$
0 \leq Q_{*} \leq \frac{1}{4} \text { and } H^{*}>\frac{1}{2}\left(1+\sqrt{1-4 Q_{*}}\right)
$$

Then system (11) is oscillatory.

Theorem 2.2. Let

$$
0 \leq H_{*} \leq \frac{1}{4} \quad \text { and } \quad Q^{*}>\frac{1}{2}\left(1+\sqrt{1-4 H_{*}}\right) .
$$

Then system (11) is oscillatory.

\section{Auxiliary proposition}

We establish some properties of solutions of equation (11) in this section. These properties are used to prove main results in what follows.

LEMma 3.1. Let $\left\{\left(u_{k}, v_{k}\right)\right\}_{k=k_{0}}^{+\infty}$ be a nonoscillatory solution of system (11). Then

$$
\sum^{\infty} R_{j}<+\infty
$$

where

$$
w_{j}=\frac{v_{j}}{u_{j}} \quad \text { and } \quad R_{j}=\frac{w_{j}^{2} q_{j}}{1+w_{j} q_{j}} .
$$




\section{ZDENĚK OPLUŠTIL}

Pr o of. We suppose on the contrary that

$$
\sum^{\infty} R_{j}=+\infty
$$

Let us put $w_{k}=\frac{v_{k}}{u_{k}}$ for $k \geq k_{0}$, then we can rewrite system (11) as follows

$$
\Delta w_{k}+p_{k}+R_{k}=0 \quad \text { for } \quad k \geq k_{0},
$$

where $R_{k}$ is defined by (77). If we summarize (9) from $k_{0}$ to $k-1$, we get

$$
-w_{k}+w_{k_{0}}=\sum_{j=k_{0}}^{k-1} p_{j}+\sum_{j=k_{0}}^{k-1} R_{j} \quad \text { for } \quad k>k_{0} .
$$

The multiplication of the last equality by $q_{k}$ and the summarization from $k_{0}$ to $k-1$ results in

$$
\sum_{j=k_{0}}^{k-1}-w_{j} q_{j}=\sum_{j=k_{0}}^{k-1} q_{j} \sum_{i=k_{0}}^{j-1} p_{i}+\sum_{j=k_{0}}^{k-1} q_{j} \sum_{i=k_{0}}^{j-1} R_{i}-w_{k_{0}} \sum_{j=k_{0}}^{k-1} q_{j} \quad \text { for } k>k_{0} .
$$

Hence,

$$
\frac{\sum_{j=k_{0}}^{k-1}-w_{j} q_{j}}{\sum_{j=k_{0}}^{k-1} q_{j}}=\frac{\sum_{j=k_{0}}^{k-1} q_{j} \sum_{i=k_{0}}^{j-1} p_{i}}{\sum_{j=k_{0}}^{k-1} q_{j}}+\frac{\sum_{j=k_{0}}^{k-1} q_{j} \sum_{i=k_{0}}^{j-1} R_{i}}{\sum_{j=k_{0}}^{k-1} q_{j}}+w_{k_{0}} \quad \text { for } k>k_{0}
$$

Consequently, in view of the assumptions (2) and (8), we get from (11)

$$
\limsup _{k \rightarrow \infty} \frac{\sum_{j=k_{0}}^{k-1}-w_{j} q_{j}}{\sum_{j=k_{0}}^{k-1} q_{j}}=+\infty .
$$

On the other hand, it is clear that

$$
R_{k}=\frac{w_{k}^{2} q_{k}}{w_{k} q_{k}+1}=\frac{w_{k}^{2}}{\frac{1}{q_{k}}+w_{k}} \geq 0 \quad \text { for } \quad k \geq k_{0} .
$$

Put

$$
A_{k}= \begin{cases}\frac{w_{k}^{2} q_{k}}{R_{k}} & \text { for } \quad k w_{k} \neq 0 \\ 0 & \text { for } k w_{k}=0\end{cases}
$$

Obviously, $A_{k} \geq 0$ for $k \geq k_{0}$ and

$$
1 \geq A_{k}-w_{k} q_{k}
$$




\section{OSCILLATORY CRITERIA FOR TWO-DIMENSIONAL SYSTEM}

The summarization of latter inequalities from $k_{0}$ to $k-1$ and (3) yield

$$
\frac{k-k_{0}}{\sum_{j=k_{0}}^{k-1} q_{j}} \geq \frac{\sum_{j=k_{0}}^{k-1} A_{j}}{\sum_{j=k_{0}}^{k-1} q_{j}}-\frac{\sum_{j=k_{0}}^{k-1} w_{j} q_{j}}{\sum_{j=k_{0}}^{k-1} q_{j}} \quad \text { for } \quad k>k_{0} .
$$

Further, the condition (3) implies

$$
\frac{k-k_{0}}{\sum_{j=k_{0}}^{k-1} q_{j}} \leq \frac{k-k_{0}}{\sum_{j=k_{0}}^{k-1} m}=\frac{1}{m} .
$$

Hence, by virtue of (15), we get

$$
\frac{1}{m} \geq \frac{\sum_{j=k_{0}}^{k-1} A_{j}}{\sum_{j=k_{0}}^{k-1} q_{j}}-\frac{\sum_{j=k_{0}}^{k-1} w_{j} q_{j}}{\sum_{j=k_{0}}^{k-1} q_{j}} \quad \text { for } \quad k, \quad k>k_{0}
$$

In view of $A_{j} \geq 0$ for $j \geq k_{0}$ the last inequality yields

$$
\limsup _{k \rightarrow \infty} \frac{\sum_{j=k_{0}}^{k-1}-w_{j} q_{j}}{\sum_{j=k_{0}}^{k-1} q_{j}}<+\infty
$$

which contradicts (12).

LEMmA 3.2. Let $0 \leq Q_{*} \leq \frac{1}{4}$ and $\left\{\left(u_{k}, v_{k}\right)\right\}_{k=k_{0}}^{+\infty}$ be a nonoscillatory solution of system (11). Then

$$
\liminf _{k \rightarrow \infty} \frac{v_{k}}{u_{k}} \sum_{j=1}^{k-1} q_{j} \geq \frac{1}{2}\left(1-\sqrt{1-4 Q_{*}}\right) .
$$

Proof. Let us put $w_{k}=\frac{v_{k}}{u_{k}}$ for $k \geq k_{0}$. The sum of (9) from $k$ to $l$ results in

$$
w_{k}-w_{l+1}=\sum_{j=k}^{l} p_{j}+\sum_{j=k}^{l} R_{j} \quad \text { for } \quad k \geq k_{0} .
$$

According to Lemma 3.1, it is clear that $\lim _{j \rightarrow \infty} w_{j}=0$. Therefore we get from (17)

$$
w_{k}=\sum_{j=k}^{\infty} p_{j}+\sum_{j=k}^{\infty} R_{j} \quad \text { for } \quad k \geq k_{0} .
$$




\section{ZDENĚK OPLUŠTIL}

Let us denote

$$
A=\liminf _{k \rightarrow \infty} w_{k} \sum_{j=1}^{k-1} q_{j}
$$

Obviously, if $A=+\infty$, then (16) holds.

Let us assume that $A<+\infty$. Now if $Q_{*}=0$, then, in view of (13) and (18), the inequality (16) holds.

Let $Q_{*}>0$. Obviously, for every $\left.\varepsilon \in\right] 0, Q_{*}\left[\right.$ there exists $k^{[\varepsilon]}>k_{0}$ such that

$$
Q_{k}>Q_{*}-\varepsilon \quad \text { for } \quad k \geq k^{[\varepsilon]} \text {. }
$$

Hence, by virtue of (18), we get

$$
w_{k} \sum_{j=1}^{k-1} q_{j}>Q_{*}-\varepsilon \quad \text { for } \quad k \geq k^{[\varepsilon]} .
$$

and $A \geq Q_{*}$. Now we can choose $k_{0}^{[\varepsilon]} \geq k^{[\varepsilon]}$ such that the inequalities

$$
w_{k} \sum_{j=1}^{k-1} q_{j} \geq A-\varepsilon, \quad\left|w_{k} q_{k}\right| \leq \varepsilon \quad \text { for } \quad k, \quad k \geq k_{0}^{[\varepsilon]}
$$

are fulfilled. Further, we can rewrite equality (18) as follows

$$
w_{k} \sum_{j=1}^{k-1} q_{j}=\sum_{j=k}^{\infty} p_{j} \sum_{j=1}^{k-1} q_{j}+\sum_{j=k}^{\infty} R_{j} \sum_{j=1}^{k-1} q_{j} \quad \text { for } \quad k, \quad k \geq k_{0} .
$$

The inequalities (20)-(22) yield

$$
w_{k} \sum_{j=1}^{k-1} q_{j} \geq Q_{*}-\varepsilon+\frac{(A-\varepsilon)^{2}}{1+\varepsilon} \quad \text { for } \quad k, \quad k \geq k_{0}^{[\varepsilon]},
$$

hence

$$
A \geq Q_{*}-\varepsilon+\frac{(A-\varepsilon)^{2}}{1+\varepsilon} .
$$

Since $\varepsilon>0$ was arbitrary we can rewrite the last inequality in the form

$$
A^{2}-A+Q_{*} \leq 0
$$

i.e.,

$$
A \geq \frac{1}{2}\left(1-\sqrt{1-4 Q_{*}}\right)
$$

Lemma 3.3. Let $0 \leq H_{*} \leq \frac{1}{4}$ and $\left\{\left(u_{k}, v_{k}\right)\right\}_{k=k_{0}}^{+\infty}$ be a nonoscillatory solution of system (1). Then

$$
\limsup _{k \rightarrow \infty} \frac{v_{k}}{u_{k}} \sum_{j=1}^{k-1} q_{j} \leq \frac{1}{2}\left(1+\sqrt{1-4 H_{*}}\right) .
$$


Proof. Let us put $w_{k}=\frac{v_{k}}{u_{k}}$ for $k \geq k_{0}$. We get from (9)

$$
\Delta w_{k}\left(\sum_{j=1}^{k} q_{j}\right)^{2}=-p_{k}\left(\sum_{j=1}^{k} q_{j}\right)^{2}-R_{k}\left(\sum_{j=1}^{k} q_{j}\right)^{2} \quad \text { for } \quad k \geq k_{0} .
$$

The summation of the last equalities from $n$ to $k-1$, where $n \geq k_{0}$, implies

$$
\sum_{j=n}^{k-1} \Delta w_{j}\left(\sum_{i=1}^{j} q_{i}\right)^{2}=-\sum_{j=n}^{k-1} p_{j}\left(\sum_{i=1}^{j} q_{i}\right)^{2}-\sum_{j=n}^{k-1} R_{j}\left(\sum_{i=1}^{j} q_{i}\right)^{2} \quad \text { for } \quad k>k_{0} .
$$

Obviously,

$$
\begin{aligned}
& \sum_{j=n}^{k-1} \Delta w_{j}\left(\sum_{i=1}^{j} q_{i}\right)^{2} \\
& =\left(\sum_{j=1}^{k-1} q_{j}\right)^{2} w_{k}-\left(\sum_{j=1}^{n-1} q_{j}\right)^{2} w_{n}-\sum_{j=n}^{k-1} w_{j} q_{j}\left(2 \sum_{i=1}^{j-1} q_{i}+q_{j}\right), \quad \text { for } \quad k>k_{0} .
\end{aligned}
$$

By virtue of (25), the equality (24) yields

$$
\begin{aligned}
\left(\sum_{j=1}^{k-1} q_{j}\right)^{2} w_{k}= & -\sum_{j=n}^{k-1} p_{j}\left(\sum_{i=1}^{j} q_{i}\right)^{2}+\left(\sum_{j=1}^{n-1} q_{j}\right)^{2} w_{n} \\
& +\sum_{j=n}^{k-1}\left[w_{j} q_{j}\left(2 \sum_{i=1}^{j-1} q_{i}+q_{j}\right)-R_{j}\left(\sum_{i=1}^{j} q_{i}\right)^{2}\right] \quad \text { for } \quad k>k_{0} .
\end{aligned}
$$

Hence

$$
w_{k}\left(\sum_{j=1}^{k-1} q_{j}\right)=-H_{k}+\frac{1}{\sum_{j=1}^{k-1} q_{j}} \sum_{j=n}^{k-1} D_{J}+P_{k, n} \quad \text { for } \quad k>k_{0},
$$

where

$$
D_{j}=w_{j} q_{j}\left(2 \sum_{i=1}^{j-1} q_{i}+q_{j}\right)-R_{j}\left(\sum_{i=1}^{j} q_{i}\right)^{2}
$$

and

$$
P_{k, n}=\frac{1}{\sum_{j=1}^{k-1} q_{j}}\left(\sum_{j=1}^{n-1} q_{j}\right)^{2} w_{n}+\frac{1}{\sum_{j=1}^{k-1} q_{j}} \sum_{j=1}^{n-1} p_{j}\left(\sum_{i=1}^{j} q_{i}\right)^{2} .
$$




\section{ZDENĚK OPLUŠTIL}

Obviously, $D_{j} \leq q_{j}$ for $j \geq k_{0}$. Thus, it follows from (26) that

$$
\left(\sum_{j=1}^{k-1} q_{j}\right) w_{k} \leq-H_{k}+1+P_{k, n} \quad \text { for } \quad k>k_{0}
$$

Further,

$$
\limsup _{k \rightarrow \infty} P_{k, n}=\limsup _{k \rightarrow \infty}\left(\frac{1}{\sum_{j=1}^{k-1} q_{j}}\left(\sum_{j=1}^{n-1} q_{j}\right)^{2} w_{n}+\frac{1}{\sum_{j=1}^{k-1} q_{j}} \sum_{j=1}^{n-1} p_{j}\left(\sum_{i=1}^{j} q_{i}\right)^{2}\right)=0 .
$$

Hence, on account of (28), we get

$$
B \leq 1-H_{*},
$$

where

$$
B=\limsup _{k \rightarrow \infty} \frac{v_{k}}{u_{k}} \sum_{j=1}^{k-1} q_{j}
$$

If $H_{*}=0$ or $B \leq 0$, then inequality (23) holds.

Let now we suppose that $H_{*}>0$ and $B>0$. We can rewrite $D_{j}$ as follows

$$
D_{j}=q_{j}\left[w_{j} \sum_{i=1}^{j-1} q_{i}\left(2-w_{j} \sum_{i=1}^{j-1} q_{i}\right)+\frac{w_{j} q_{j}}{1+w_{j} q_{j}}\left(w_{j} \sum_{i=1}^{j-1} q_{i}-1\right)^{2}\right] \text { for } k, j \geq k_{0}
$$

then (26) transforms into

$$
\begin{aligned}
& \left(\sum_{j=1}^{k-1} q_{j}\right) w_{k}=-H_{k}+P_{k, n} \\
& -\frac{1}{\sum_{j=1}^{k-1} q_{j}} \sum_{j=n}^{k-1}\left[q_{j}\left(w_{j} \sum_{i=1}^{j-1} q_{i}\left(2-w_{j} \sum_{i=1}^{j-1} q_{i}\right)+\frac{w_{j} q_{j}}{1+w_{j} q_{j}}\left(w_{j} \sum_{i=1}^{j-1} q_{i}-1\right)^{2}\right)\right] .
\end{aligned}
$$

Let $0<\varepsilon<\min \left\{H_{*}, 1-B\right\}$ be arbitrary. We can choose $k^{[\varepsilon]}>k_{0}$ such that

$$
\sum_{j=1}^{k-1} q_{j} w_{j}<B+\varepsilon, \quad H_{k}>H_{*}-\varepsilon \quad \text { and } \quad\left|\frac{q_{k} w_{k}}{1+w_{k}}\right| \leq \varepsilon \quad \text { for } \quad k \geq k^{[\varepsilon]} .
$$

Since the function $f(x)=x(2-x)+\varepsilon(x-1)^{2}$ is nondecreasing in $] \varepsilon, 1[$ and $B+\varepsilon \in] \varepsilon, 1[$, it follows from (31) and (32) that 


$$
w_{k} \sum_{j=1}^{k-1} q_{j} \leq-H_{*}+\varepsilon+\left[(B+\varepsilon)(2-B-\varepsilon)+\varepsilon(B+\varepsilon-1)^{2}\right] \frac{\sum_{j=k^{[\varepsilon]}}^{k-1} q_{j}}{\sum_{j=1}^{k-1} q_{j}}+P_{k, k^{[\varepsilon]}} .
$$

Hence, by virtue of (29) and (30), we get

$$
B \leq-H_{*}+\varepsilon+\left[(B-\varepsilon)(2-B-\varepsilon)+\varepsilon(B+\varepsilon-1)^{2}\right] .
$$

Since $\varepsilon>0$ was chosen arbitrary we have

$$
B^{2}-B+H_{*} \leq 0 .
$$

Obviously, latter inequality implies

$$
B \leq \frac{1}{2}\left(1+\sqrt{1-4 H_{*}}\right)
$$

\section{Proofs of main results}

Proof of Theorem 2.1. Let us assume on the contrary, that system (1) is nonoscillatory, i.e., there exists a solution $\left\{\left(u_{k}, v_{k}\right)\right\}_{k=k_{0}}^{+\infty}$ such that

$$
u_{k} u_{k+1}>0 \text { for } k \geq k_{0} .
$$

Now we can rewrite system (1) in the form

$$
\Delta w_{k}+p_{k}+R_{k}=0 \quad \text { for } \quad k \geq k_{0}
$$

where

$$
w_{k}=\frac{v_{k}}{u_{k}} \quad \text { and } \quad R_{k}=\frac{w_{k}^{2} q_{k}}{1+w_{k} q_{k}} \quad \text { for } \quad k \geq k_{0} .
$$

Let us denote

$$
\tilde{A}=\frac{1}{2}\left(1-\sqrt{1-4 Q_{*}}\right) .
$$

According to Lemma 3.2 there exists $k^{[\varepsilon]}>k_{0}$ such that

$$
w_{k} \sum_{j=1}^{k-1} q_{j}>\tilde{A}-\varepsilon \quad \text { for } \quad k \geq k^{[\varepsilon]}
$$

for arbitrary $\varepsilon>0$. Now we can show, in the similar way as in the proof of Lemma 3.3. that (26) and inequality $D_{j} \leq q_{j}$ for $k \geq k^{[\varepsilon]}$ hold. Thus,

$$
w_{k} \sum_{j=1}^{k-1} q_{j} \leq-H_{k}+1+P_{k, k}[\varepsilon] \quad \text { for } \quad k \geq k^{[\varepsilon]} .
$$




\section{ZDENĚK OPLUŠTIL}

On account of (34), the latter inequality yields

i.e.,

$$
H_{k} \leq-\tilde{A}+\varepsilon+1+P_{k, k[\varepsilon]} \quad \text { for } k \geq k^{[\varepsilon]},
$$

$$
H_{k} \leq-\frac{1}{2}\left(1-\sqrt{1-4 Q_{*}}\right)+\varepsilon+1+P_{k, k[\varepsilon]} \quad \text { for } \quad k \geq k^{[\varepsilon]} .
$$

Hence

$$
H^{*} \leq \frac{1}{2}\left(1+\sqrt{1-4 Q_{*}}\right)+\varepsilon
$$

which contradicts (4), since $\varepsilon>0$ was chosen arbitrary.

Pro of of The or em 2.2. Let us assume on the contrary, that system (10) is nonoscillatory, i.e., there exists a solution $\left\{\left(u_{k}, v_{k}\right)\right\}_{k=k_{0}}^{+\infty}$ such that

$$
u_{k} u_{k+1}>0 \quad \text { for } \quad k \geq k_{0} \text {. }
$$

Now we can rewrite system (11) in the form

$$
\Delta w_{k}+p_{k}+R_{k}=0 \quad \text { for } k \geq k_{0},
$$

where

$$
w_{k}=\frac{v_{k}}{u_{k}} \quad \text { and } \quad R_{k}=\frac{w_{k}^{2} q_{k}}{1+w_{k} q_{k}} \quad \text { for } \quad k \geq k_{0} .
$$

Put

$$
\tilde{B}=\frac{1}{2}\left(1+\sqrt{1-4 H_{*}}\right) .
$$

According to Lemma 3.3 there exists $k^{[\varepsilon]}>k_{0}$ such that

$$
\tilde{B}+\varepsilon>w_{k} \sum_{j=1}^{k-1} q_{j} \quad \text { for } \quad k \geq k^{[\varepsilon]}
$$

for arbitrary $\varepsilon>0$. Analogously as in proof of Lemma 3.2 we can prove that the inequality (22) for $k \geq k^{[\varepsilon]}$ holds. Thus, it follows from (35) that

$$
\tilde{B}+\varepsilon>\sum_{j=1}^{k-1} q_{j} \sum_{j=k}^{\infty} p_{j}+\sum_{j=1}^{k-1} q_{j} \sum_{j=k}^{\infty} R_{j} \quad \text { for } \quad k \geq k^{[\varepsilon]} .
$$

By virtue of (3) and (7), the latter inequality implies

$$
\frac{1}{2}\left(1+\sqrt{1-4 H_{*}}\right)+\varepsilon>Q_{k} \quad \text { for } \quad k \geq k^{[\varepsilon]},
$$

which contradicts (5), since $\varepsilon>0$ was chosen arbitrary. 


\title{
OSCILLATORY CRITERIA FOR TWO-DIMENSIONAL SYSTEM
}

\section{REFERENCES}

[1] AGARWAL, R. P.: Difference Equations and Inequalities, Theory, Methods and Applications, in: Pure Appl. Math. (N.Y.), M. Dekker, New York, 1992.

[2] GRAEF, J. R.-THANDAPANI, E.: Oscillation of two-dimensional difference system, Comput. Math. Appl. 38 (1999), 157-165.

[3] HILlE, E.: Nonoscillation theorems, Trans. Amer. Math. Soc. 64 (1948), 234-252.

[4] CHANTLADZE, T.-KANDELAKI, N.-LOMTATIDZE, A.: Oscillation and nonoscillation criteria for a second order linear equation, Georgian Math. J. 6 (1999), 401-414.

[5] LOMTATIDZE, A.: Oscillation and nonoscillation criteria for second order linear differential equation, Georgian Math. J. 4 (1997), 129-138.

[6] NEHARI, Z.: Oscillation criteria for seconder-order linear differential equations, Trans. Amer. Math. Soc. 85 (1957), 428-445.

[7] SZAFRANSKI, Z.-SZMANDA, B.: Oscillatory properties of solutions of some difference systems, Rad. Mat. 2 (1990), 205-214.

[8] THANDAPANI, E.-PONNAMMAL, B.: On the oscillation of a nonlinear two-dimensional difference system, Tamkang J. Math. 32 (2001), 201-209.

[9] THANDAPANI, E.-PONNAMMAL, B.: Oscillatory and asymptotic behavior of solutions of nonlinear two-dimensional difference systems, Math. Sci. Res. Hot-Line 4 (2000), $1-18$.

[10] WINTNER, A.: A criterion of oscillatory stability, Quart. Appl. Math. 7 (1949), 115-117.

Received November 15, 2010

\author{
Institute of Mathematics \\ Faculty of Mechanical Engineering \\ Technická 2 \\ CZ-616-69 Brno \\ CZECH REPUBLIC \\ E-mail: oplustil@fme.vutbr.cz
}

